\title{
SPOTTED
}

\section{Spotted around the web: PTEN organoids, virtual reality, decolonizing science}

BY JILL ADAMS, LAURA DATTARO, PETER HESS, NIKO MCCARTY

10 SEPTEMBER 2021 